\title{
Statin Use Is Associated With Reduced Mortality On Mechanically Ventilated Patients:A Retrospective Propensity-Matched Analysis
}

\section{Daonan Chen}

Emergency and Critical Care, Shanghai General hospital of Shanghai Jiaotong Univerisity

\section{Liu Wang}

Emergency and Critical Care, Shanghai General Hospital of Shanghai Jiaotong University

Huifang Zhang

Emergency and Critical Care, Shanghai General Hospital of Shanghai Jiaotong University

Qiuhai Lin

Emergency and Critical Care, Shanghai General Hospital of Shanghai Jiaotong University

\section{Rui Tian}

Emergency and Critical Care, Shanghai General Hospital of Shanghai Jiaotong University Jiyao Xu

Emergency and Critical Care, Shanghai General Hospital of Shanghai Jiaotong University

\section{Congliang Miao}

Emergency and Critical Care, Shanghai General Hospital of Shanghai Jiaotong University

\section{Yuan Huang}

Emergency and Critical Care, Shanghai General Hospital of Shanghai Jiaotong University

\section{Fangxia Ge}

Emergency and Critical Care, Shanghai General Hospital of Shanghai Jiaotong University

\section{Mei Kang}

Clinical Research Center, Shanghai General Hospital of Shanghai Jiaotong University

\section{Yong Zhu}

Emergency and Critical Care, Shanghai General Hospital of Shanghai Jiaotong University

\section{Yun Xie}

Emergency and Critical Care, Shanghai General Hospital of Shanghai Jiaotong University

\section{Ruilan Wang}

Emergency and Critical Care, Shanghai General Hospital of Shanghai Jiaotong University

Jiang Du ( $\backsim$ gowindj@163.com )

Shanghai Jiaotong University Children's Hospital: Children's Hospital of Shanghai

https://orcid.org/0000-0002-8297-5359 
Keywords: Statin, Mechanical Ventilation, Lung Injury, MIMIC, Big Data

Posted Date: April 12th, 2021

DOI: https://doi.org/10.21203/rs.3.rs-383754/v1

License: (c) (i) This work is licensed under a Creative Commons Attribution 4.0 International License. Read Full License 


\section{Abstract}

\section{Background}

The patients on mechanical ventilation were always critically ill and ventilation may cause injury and inflammatory disorders in the lungs of such patients. We sought to determine whether statin treatment has a protective effect on the outcome of the ventilated patients.

\section{Methods}

A retrospective observational study of mechanically ventilated patients from the Medical Information Mart for Intensive Care III (MIMIC-III) database. To ensure the robustness of the findings, we utilized the gradient boosted model, propensity score analysis, doubly robust estimation and an inverse probabilityweighting model in the statistical procedure. The propensity score of statin use was calculated by age, sex, severity scores and 32 other variables.

\section{Results}

Compared with nonusers, the use of statins was associated with improved 28-day survival in the unmatched cohort (HR $0.8595 \% \mathrm{Cl} 0.80 \sim 0.90, \mathrm{p}<0.01)$ and matched cohort (HR $0.7995 \% \mathrm{Cl} 0.73 \sim 0.85$, $\mathrm{p}<0.01)$. Statin use was also associated with improved 60-day (HR $0.8195 \% \mathrm{Cl} 0.75 \sim 0.85, \mathrm{p}<0.01), 90$ day (HR $0.8195 \% \mathrm{Cl} 0.76 \sim 0.86, p<0.01)$ and in-hospital (HR $0.8195 \% \mathrm{Cl} 0.76 \sim 0.86, p<0.01)$ survival in the matched cohort. Statins use was associated with longer ventilator free days (VFD28, 14.93 $\pm 13.11 \mathrm{vs}$ $12.06 \pm 13.26, p<0.01)$ and longer ICU free days $(13.41 \pm 12.14$ vs $10.86 \pm 12.19, p<0.01)$ in the matched cohort. Different types of statins were associated with improved 28-day survival. The subgroup analyses results showed improved 28-day survival in most subgroups, but this improvement was not observed in patients with pneumonia, septicemia or acute respiratory failure.

\section{Conclusions}

In a population of mechanically ventilated patients, the use of statins may be associated with reduced mortality. This protective effect was observed only in mild-to-moderate patients instead of critical inflammatory ones.

Trial registration

http://www.chictr.org.cn/index.aspx; Registration number: ChiCTR2000029594

\section{Background}

Statins, also known as 3-hydroxy-3 methylblycel coenzyme A (HMG-CoA) reductase inhibitors, exert pleiotropic effects in addition to their lipid-lowering effects in the context of coronary artery disease [1] and ischemic stroke [2] prophylaxes. Studies in vitro and in vivo have shown that statins can provide 
additional protective effects, including the reduction in inflammation, immunomodulation, antimicrobial effects, improved endothelial cell function and antithrombotic actions [3-7]. In pneumonia patients, current statin use was associated with decreased mortality [8]. Statin use or prior use may have decreased the mortality of septic patients in some observational studies [9-11]. Preliminary data from animal models have shown the protective effect on lung injury induced by sepsis [12]. The results of the meta-analysis support a similar conclusion[13]. However, some randomized controlled studies have come to the opposite conclusion[14-18]. No benefit of statin therapy in patients were shown in patients with sepsis or septic shock. and this was supported by two recent meta-analysis[19, 20].

The differences in outcomes between the studies might be attributed to the characteristics of different ICU populations. One recent retrospective study enrolled all the ICU patients who had been prescribed statins and compared the outcome with a matching cohort. The result showed a beneficial association of statin use and 90-day mortality improvement [21]. However, the ICU population in this study may have been healthier or more self-limiting disease processes than other ICU populations, such as patients with sepsis or acute lung injury/ARDS; this could have resulted in the relatively lower 90-day mortality observed in this study compared to that reported in other studies. To avoid such patient selection bias and to, moreover, research on the mortality benefit of statin use in the critically ill, we enrolled ventilated patients who had taken statin before or during ventilation as our study population. Ventilation is a key life-saving treatment measure for critically ill patients, including patients with acute respiratory distress syndrome (ARDS), trauma, shock and other life-threatening conditions. Epidemiological data have shown that there were an estimated 790,257 hospitalizations involving mechanical ventilation in 2005 in the USA, representing 2.7 cases of mechanical ventilation per 1000 people. The estimated national associated cost was $\$ 27$ billion, representing $12 \%$ of hospital costs, which accounts for a large amount of resources in the critical care department [22]. Furthermore, ventilation can cause lung injury and lead to ventilator-associated pneumonia (VAP) and other severe complications, which may increase the mortality of critically ill patients [23]. Lung injury remains one of the major complications of mechanical ventilation in the intensive care unit (ICU).

Whether statin use is associated with a lower mortality in critically ill patients requiring ventilation in the intensive care unit (ICU) remains unclear. Therefore, we designed this observational study to research the potential beneficial effect of statin use among critically ill ventilated patients.

\section{Methods}

\section{Study design and data source}

This is a retrospective observational study. We analyzed data from a large database: Medical Information Mart for Intensive Care (MIMIC-III). The MIMIC-III database is an openly available dataset developed by the MIT Laboratory for Computational Physiology, comprising deidentified health data associated with nearly 54,000 intensive care unit admissions [24]. The data in the MIMIC-III database are composed of comprehensive clinical datasets from patients admitted to the ICUs of Beth Israel Deaconess Medical 
Center in Boston, MA. The requirement for institutional review board (IRB) approval from our institution was exempted because MIMIC-III is a third-party anonymized publicly available database with preexisting IRB approval.

\section{Participants}

Patients who underwent mechanical ventilation were selected from the MIMIC-III database. The ventilation data were extracted from the chartvcents table. Statin usage information was extracted from the prescription table. We selected those who had taken statins before or during ventilation as the statin cohort and those who underwent ventilation without statins as the control cohort. Those who took statin medicine after extubation were excluded from this study. We included only adults in this study, so those under 18 years old were excluded. A total of 3999 patients were selected for the statin cohort. Then, we performed a propensity matching by age, sex, Simplified Acute Physiology Score II (SAPSII), and 32 other variables. The detail propensity score calculation was explained in the statistics part later. Each statinexposed patient was matched with the closest corresponding non-exposed patient (that is, a patient who was not exposed to statins) at a 1:1 fixed ratio (nearest match cohort). Finally, 3363 patients were matched and included in each cohort.

\section{Medication exposure}

Patients who had taken statins before or during ventilation were selected as the statin cohort, and those who underwent ventilation without statins were selected as the control cohort. Patients taking statin medicine after extubation were excluded from this study. Atorvastatin, pravastatin, rosuvastatin and simvastatin were the 4 most common statin types. A total of 187 patients received atorvastatin plus simvastatin.

\section{Outcomes}

The primary outcome was 28-day and in-hospital all-cause mortality. The primary statistical method of comparison for the time-to-event end points was expressed by the Kaplan-Meier curves and tested by the log-rank test. A Cox proportional hazards model was used to estimate the hazard ratio (HR) of 28-day mortality and its associated $95 \%$ confidence interval $(\mathrm{Cl})$. We included several variables in the model to adjust the 28-day survival (age, SAPSII score, sex, liver disease, diabetes, obesity, hypertension, etc.). The APACHE III score was not recorded for every patient, so we could not extract all APACHE III scores. The SAPSII was chosen to represent the severity of illness. The secondary outcome analyses also included ICU free days in 28-day, ventilator free days in 28 days, 60-day survival, 90-day survival and in hospital survival. Eight plasma biomarkers were extracted and studied to measure the host responses during the first 10 days after intubation. The HRs of each type of statins were studied by COX models. The effect of statin in different subgroup population were analyzed in the end.

\section{Statistical methods}

The doubly robust estimation method was applied to infer the sensitivity analysis of the primary outcome. "Doubly robust estimation combines a multivariate regression model with a propensity score 
model to estimate the association and causal effect of an exposure on an outcome" [25, 26]. Usually, the regression model or the propensity score model was applied individually to estimate a causal effect. When the two approaches were built in one estimation model, only one of the two models needs to be correctly specified to obtain an unbiased effect estimator, thus the term doubly robust analysis. For propensity scores of the statin use, a machine learning algorithm named gradient boosted model (GBM) was employed to maximally correlated with the negative gradient of the predefined loss function. Regression tree was used and a total of 35 covariates were used in the model so that covariate imbalance between the statin and non-statin groups was minimized. Using the estimated propensity scores calculated by GBM model as weights, an inverse probability weighting (IPW) model was used to generate a weighted cohort $[26,27]$. An IPW weighted COX regression was then built adjusting for the variables that remained unbalanced between the groups. The propensity score was also employed to build the 1:1 matching cohort of the non-statin cohort.

Some studies have reported different potencies between statins; for example, simvastatin exerted better antibacterial effects than rosuvastatin, and the latter was found to have a more potent lipid-lowering capacity $[28,29]$. Therefore, we analyzed the difference in 28-day survival among statin types in the Cox regression model. To test the efficiency of statins on patients with different profiles, we performed a subgroup analysis with age and SAPSII as covariates. Patient categorical data are presented as percentages, and continuous data are listed as means with standard deviations (SDs). We used Student's $t$ tests for continuous variables and chi-square or Fisher's exact tests for dichotomous variables. Rstudio1.2.1335 (RStudio, Inc., Boston, MA, USA) were used to perform the statistical analyses.

\section{Results}

\section{Baseline results}

All 53432 cases in the MIMIC-III database were screened. A total of 24,769 individuals who had undergone ventilation met the inclusion criteria. A total of 17065 patients who had not received statin treatment were included in the non-statin cohort. We constructed a propensity score model by employing the 39 covariates with the GBM. The contributions of individual covariates to the final propensity score are illustrated in Fig. 1. The top covariates include diagnosis, age, history of hypertension, presence of $\mathrm{CHF}$ and peripheral vascular disease: such factors would commonly influence the decision regarding whether to prescribe statin. Based on the estimated propensity scores, IPW was applied to standardize the differences between the statin and non-statin cohorts. As shown in Table 1, most of the covariates of the weighted cohorts were balanced between the two cohorts with or without statin. There were 3999 patients who received statin treatment before or during the ventilation time. After matching, there were 3363 patients in each cohort (Table 1). They were similarities in age, sex, SAPSII and 36 more variables. The characteristics of the patients are presented in Table 1. There were 2145 cases of atorvastatin, 200 pravastatin, 171 rosuvastatin, 1284 simvastatin and 199 cases received other statins in the unmatched cohort. In the matched cohort, there were 1835 cases of atorvastatin, 162 pravastatin, 136 rosuvastatin, 1068 simvastatin and 162 cases received other statins. 
Table 1

Demographic data

\begin{tabular}{|c|c|c|c|c|c|c|c|}
\hline & $\begin{array}{l}\text { statin } \\
\text { unmatched }\end{array}$ & $\begin{array}{l}\text { Non-statin } \\
\text { unmatched }\end{array}$ & $\begin{array}{l}\text { p- } \\
\text { value }\end{array}$ & SMD & $\begin{array}{l}\text { statin } \\
\text { matched }\end{array}$ & $\begin{array}{l}\text { Non-statin } \\
\text { matched }\end{array}$ & SMD2 \\
\hline $\mathrm{n}$ & 3999 & 17452 & & & 3363 & 3363 & \\
\hline $\begin{array}{l}\text { Age* (mean } \\
(\mathrm{SD}))\end{array}$ & $\begin{array}{l}69.96 \\
(11.92)\end{array}$ & $\begin{array}{l}62.06 \\
(17.63)\end{array}$ & $<0.001$ & 0.525 & $\begin{array}{l}70.09 \\
(12.15)\end{array}$ & $\begin{array}{l}70.37 \\
(11.73)\end{array}$ & 0.023 \\
\hline $\begin{array}{l}\text { oasis (mean } \\
\text { (SD)) }\end{array}$ & $\begin{array}{l}35.59 \\
(8.22)\end{array}$ & 36.61 (8.39) & $<0.001$ & 0.124 & $\begin{array}{l}35.89 \\
(8.32)\end{array}$ & $\begin{array}{l}36.29 \\
(8.03)\end{array}$ & 0.049 \\
\hline $\begin{array}{l}\text { sofa (mean } \\
(\text { SD)) }\end{array}$ & $5.35(3.02)$ & $5.31(3.57)$ & 0.554 & 0.011 & $\begin{array}{l}5.25 \\
(3.04)\end{array}$ & $\begin{array}{l}5.38 \\
(3.03)\end{array}$ & 0.044 \\
\hline $\begin{array}{l}\text { Sapsii* (mean } \\
\text { (SD)) }\end{array}$ & $\begin{array}{l}41.04 \\
(13.39)\end{array}$ & $\begin{array}{l}39.73 \\
(15.68)\end{array}$ & $<0.001$ & 0.089 & $\begin{array}{l}40.96 \\
(13.46)\end{array}$ & $\begin{array}{l}40.91 \\
(13.16)\end{array}$ & 0.004 \\
\hline Gender* = M (\%) & $\begin{array}{l}2485 \\
(62.1)\end{array}$ & 9967 ( 57.1) & $<.001$ & 0.103 & $\begin{array}{l}2030 \\
(60.4)\end{array}$ & $\begin{array}{l}2033 \\
(60.5)\end{array}$ & 0.002 \\
\hline statin type (\%) & & & $\begin{array}{l}< \\
0.001\end{array}$ & 1.315 & & & 1.923 \\
\hline Atorvastatin & $\begin{array}{l}2145 \\
(53.6)\end{array}$ & $0(0.0)$ & & & $\begin{array}{l}1835 \\
(54.6)\end{array}$ & $0(0.0)$ & \\
\hline Non user & $0(0.0)$ & $\begin{array}{l}17452 \\
(100.0)\end{array}$ & & & $0(0)$ & $3363(100)$ & \\
\hline Other statins & 199 ( 5.0) & $0(0.0)$ & & & $\begin{array}{l}162 \\
(4.8)\end{array}$ & $0(0.0)$ & \\
\hline Pravastatin & $200(5.0)$ & $0(0.0)$ & & & $\begin{array}{l}136 \\
(4.0)\end{array}$ & $0(0.0)$ & \\
\hline Rosuvastatin & $171(4.3)$ & $0(0.0)$ & & & $\begin{array}{l}1068 \\
(31.8)\end{array}$ & $0(0.0)$ & \\
\hline Simvastatin & $\begin{array}{l}1284 \\
(32.1)\end{array}$ & $0(0.0)$ & & & $\begin{array}{l}162 \\
(4.8)\end{array}$ & $0(0.0)$ & \\
\hline \multicolumn{8}{|l|}{ Commobidities* } \\
\hline $\begin{array}{l}\text { congestive heart } \\
\text { failure (\%) }\end{array}$ & $\begin{array}{l}1718 \\
(43.0)\end{array}$ & 4573 ( 26.2) & $<0.001$ & 0.358 & $\begin{array}{l}1430 \\
(42.5)\end{array}$ & $\begin{array}{l}1481 \\
(44.0)\end{array}$ & 0.031 \\
\hline
\end{tabular}

Continuous variables are presented as mean (standard deviation), categorical as frequency (percentage). T-test was used to compare statin recipients vs non-statin for continuous variables, Fisher's exact test for categorical variables. Standardized differences (SD) are defined as the difference in means, proportions or ranks divided by the mutual standard deviation. ${ }^{*}$ ) variables were used for the calculation of propensity scores.Abbreviations:LOS: length of stay; OASIS: Oxford Acute Severity of IIIness Score; SOFA: Sequential Organ Failure Assessment; SAPSII: Simplified Acute Physiology Score II. 


\begin{tabular}{|c|c|c|c|c|c|c|c|}
\hline & $\begin{array}{l}\text { statin } \\
\text { unmatched }\end{array}$ & $\begin{array}{l}\text { Non-statin } \\
\text { unmatched }\end{array}$ & $\begin{array}{l}\mathrm{p}- \\
\text { value }\end{array}$ & SMD & $\begin{array}{l}\text { statin } \\
\text { matched }\end{array}$ & $\begin{array}{l}\text { Non-statin } \\
\text { matched }\end{array}$ & SMD2 \\
\hline $\begin{array}{l}\text { cardiac } \\
\text { arrhythmias (\%) }\end{array}$ & $\begin{array}{l}1849 \\
(46.2)\end{array}$ & $5011(28.7)$ & $<.001$ & 0.368 & $\begin{array}{l}1518 \\
(45.1)\end{array}$ & $\begin{array}{l}1537 \\
(45.7)\end{array}$ & 0.011 \\
\hline $\begin{array}{l}\text { valvular disease } \\
\text { (\%) }\end{array}$ & $\begin{array}{l}1251 \\
(31.3)\end{array}$ & $2561(14.7)$ & $<0.001$ & 0.403 & $\begin{array}{l}981 \\
(29.2)\end{array}$ & $\begin{array}{l}1010 \\
(30.0)\end{array}$ & 0.019 \\
\hline $\begin{array}{l}\text { pulmonary } \\
\text { circulation (\%) }\end{array}$ & 395 ( 9.9) & $1253(7.2)$ & $<.001$ & 0.097 & $\begin{array}{l}317 \\
(9.4)\end{array}$ & $311(9.2)$ & 0.006 \\
\hline $\begin{array}{l}\text { peripheral } \\
\text { vascular (\%) }\end{array}$ & 775 (19.4) & 1659 (9.5) & $<.001$ & 0.284 & $\begin{array}{l}584 \\
(17.4)\end{array}$ & $617(18.3)$ & 0.026 \\
\hline $\begin{array}{l}\text { other } \\
\text { neurological (\%) }\end{array}$ & $482(12.1)$ & $2551(14.6)$ & $<.001$ & 0.075 & $\begin{array}{l}419 \\
(12.5)\end{array}$ & 416 (12.4) & 0.003 \\
\hline $\begin{array}{l}\text { chronic } \\
\text { pulmonary (\%) }\end{array}$ & $962(24.1)$ & 3629 ( 20.8) & $<.001$ & 0.078 & $\begin{array}{l}830 \\
(24.7)\end{array}$ & $841(25.0)$ & 0.008 \\
\hline $\begin{array}{l}\text { diabetes } \\
\text { uncomplicated } \\
(\%)\end{array}$ & $\begin{array}{l}1195 \\
(29.9)\end{array}$ & 3184 ( 18.2) & $\begin{array}{l}< \\
0.001\end{array}$ & 0.275 & $\begin{array}{l}954 \\
(28.4)\end{array}$ & $977(29.1)$ & 0.015 \\
\hline $\begin{array}{l}\text { diabetes } \\
\text { complicated (\%) }\end{array}$ & 415 (10.4) & $922(5.3)$ & $<.001$ & 0.190 & $\begin{array}{l}312 \\
(9.3)\end{array}$ & $337(10.0)$ & 0.025 \\
\hline hypertension (\%) & $\begin{array}{l}2949 \\
(73.7)\end{array}$ & 8219 ( 47.1) & $<.001$ & 0.566 & $\begin{array}{l}2353 \\
(70.0)\end{array}$ & $\begin{array}{l}2397 \\
(71.3)\end{array}$ & 0.029 \\
\hline paralysis (\%) & $193(4.8)$ & 735 ( 4.2) & 0.093 & 0.030 & $\begin{array}{l}169 \\
(5.0)\end{array}$ & $167(5.0)$ & 0.003 \\
\hline $\begin{array}{l}\text { hypothyroidism } \\
\text { (\%) }\end{array}$ & $469(11.7)$ & $1538(8.8)$ & $<.001$ & 0.096 & $\begin{array}{l}387 \\
(11.5)\end{array}$ & $364(10.8)$ & 0.022 \\
\hline renal failure (\%) & $800(20.0)$ & $2112(12.1)$ & $\begin{array}{l}<.001 \\
0.001\end{array}$ & 0.217 & $\begin{array}{l}617 \\
(18.3)\end{array}$ & $629(18.7)$ & 0.009 \\
\hline liver disease (\%) & 108 ( 2.7) & $1843(10.6)$ & $<.001$ & 0.320 & $97(2.9)$ & $90(2.7)$ & 0.013 \\
\hline peptic ulcer (\%) & $1(0.0)$ & $16(0.1)$ & 0.298 & 0.028 & $1(0.0)$ & $0(0.0)$ & 0.024 \\
\hline lymphoma (\%) & $39(1.0)$ & 339 ( 1.9) & $\begin{array}{l}< \\
0.001\end{array}$ & 0.081 & $32(1.0)$ & $36(1.1)$ & 0.012 \\
\hline $\begin{array}{l}\text { metastatic } \\
\text { cancer (\%) }\end{array}$ & 78 ( 2.0) & $1092(6.3)$ & $<.001$ & 0.218 & $78(2.3)$ & $80(2.4)$ & 0.004 \\
\hline \multicolumn{8}{|c|}{$\begin{array}{l}\text { Continuous variables are presented as mean (standard deviation), categorical as frequency } \\
\text { (percentage). T-test was used to compare statin recipients vs non-statin for continuous variables, } \\
\text { Fisher' s exact test for categorical variables. Standardized differences (SD) are defined as the } \\
\text { difference in means, proportions or ranks divided by the mutual standard deviation. (*)variables were } \\
\text { used for the calculation of propensity scores.Abbreviations:LOS: length of stay; OASIS: Oxford Acute } \\
\text { Severity of Illness Score; SOFA: Sequential Organ Failure Assessment; SAPSII: Simplified Acute } \\
\text { Physiology Score II. }\end{array}$} \\
\hline
\end{tabular}




\begin{tabular}{|c|c|c|c|c|c|c|c|}
\hline & $\begin{array}{l}\text { statin } \\
\text { unmatched }\end{array}$ & $\begin{array}{l}\text { Non-statin } \\
\text { unmatched }\end{array}$ & $\begin{array}{l}\mathrm{p}- \\
\text { value }\end{array}$ & SMD & $\begin{array}{l}\text { statin } \\
\text { matched }\end{array}$ & $\begin{array}{l}\text { Non-statin } \\
\text { matched }\end{array}$ & SMD2 \\
\hline solid tumor (\%) & $111(2.8)$ & 990 ( 5.7) & $<.001$ & 0.144 & $\begin{array}{l}102 \\
(3.0)\end{array}$ & $101(3.0)$ & 0.002 \\
\hline $\begin{array}{l}\text { Theumatoid } \\
\text { arthritis (\%) }\end{array}$ & 103 ( 2.6) & 459 ( 2.6) & 0.889 & 0.003 & $90(2.7)$ & $86(2.6)$ & 0.007 \\
\hline $\begin{array}{l}\text { coagulopathy } \\
\text { (\%) }\end{array}$ & $520(13.0)$ & 2499 ( 14.3) & 0.033 & 0.038 & $\begin{array}{l}418 \\
(12.4)\end{array}$ & 400 (11.9) & 0.016 \\
\hline obesity (\%) & $448(11.2)$ & $1033(5.9)$ & $<.001$ & 0.190 & $\begin{array}{l}297 \\
(8.8)\end{array}$ & 311 (9.2) & 0.015 \\
\hline weight loss (\%) & $122(3.1)$ & $957(5.5)$ & $\begin{array}{l}< \\
0.001\end{array}$ & 0.121 & $\begin{array}{l}114 \\
(3.4)\end{array}$ & $104(3.1)$ & 0.017 \\
\hline $\begin{array}{l}\text { fluid electrolyte } \\
\text { (\%) }\end{array}$ & $\begin{array}{l}1230 \\
(30.8)\end{array}$ & 5840 ( 33.5) & 0.001 & 0.058 & $\begin{array}{l}1035 \\
(30.8)\end{array}$ & $\begin{array}{l}1012 \\
(30.1)\end{array}$ & 0.015 \\
\hline $\begin{array}{l}\text { blood loss } \\
\text { anemia (\%) }\end{array}$ & $60(1.5)$ & $353(2.0)$ & 0.035 & 0.040 & $54(1.6)$ & $42(1.2)$ & 0.030 \\
\hline $\begin{array}{l}\text { deficiency } \\
\text { anemias (\%) }\end{array}$ & $968(24.2)$ & 2907 ( 16.7) & $<.001$ & 0.188 & $\begin{array}{l}681 \\
(20.2)\end{array}$ & $690(20.5)$ & 0.007 \\
\hline $\begin{array}{l}\text { alcohol abuse } \\
\text { (\%) }\end{array}$ & 135 ( 3.4$)$ & $1732(9.9)$ & $<.001$ & 0.265 & $\begin{array}{l}117 \\
(3.5)\end{array}$ & 105 (3.1) & 0.020 \\
\hline drug abuse (\%) & $50(1.3)$ & 803 ( 4.6) & $\begin{array}{l}< \\
0.001\end{array}$ & 0.200 & $47(1.4)$ & $35(1.0)$ & 0.033 \\
\hline psychoses (\%) & $126(3.2)$ & 756 ( 4.3) & 0.001 & 0.062 & $\begin{array}{l}100 \\
(3.0)\end{array}$ & $92(2.7)$ & 0.014 \\
\hline depression (\%) & 315 ( 7.9) & $1354(7.8)$ & 0.826 & 0.004 & $\begin{array}{l}234 \\
(7.0)\end{array}$ & $216(6.4)$ & 0.021 \\
\hline Diagnosis* (\%) & & & $<.001$ & 0.958 & & & 0.112 \\
\hline $\begin{array}{l}\text { Benign } \\
\text { Neoplasm }\end{array}$ & $9(0.2)$ & $97(0.6)$ & & & $9(0.3)$ & $7(0.2)$ & \\
\hline Bone Fracture & $68(1.7)$ & $855(4.9)$ & & & $68(2.0)$ & $54(1.6)$ & \\
\hline $\begin{array}{l}\text { Cerebral } \\
\text { infarction }\end{array}$ & 129 ( 3.2$)$ & $206(1.2)$ & & & $\begin{array}{l}113 \\
(3.4)\end{array}$ & $121(3.6)$ & \\
\hline
\end{tabular}

Continuous variables are presented as mean (standard deviation), categorical as frequency (percentage). T-test was used to compare statin recipients vs non-statin for continuous variables, Fisher's exact test for categorical variables. Standardized differences (SD) are defined as the difference in means, proportions or ranks divided by the mutual standard deviation. ${ }^{*}$ ) variables were used for the calculation of propensity scores.Abbreviations:LOS: length of stay; OASIS: Oxford Acute Severity of IIIness Score; SOFA: Sequential Organ Failure Assessment; SAPSII: Simplified Acute Physiology Score II. 


\begin{tabular}{|c|c|c|c|c|c|c|}
\hline & $\begin{array}{l}\text { statin } \\
\text { unmatched }\end{array}$ & $\begin{array}{l}\text { Non-statin } \\
\text { unmatched }\end{array}$ & $\begin{array}{l}\mathrm{p}- \\
\text { value }\end{array}$ & SMD & $\begin{array}{l}\text { statin } \\
\text { matched }\end{array}$ & $\begin{array}{l}\text { Non-statin } \\
\text { matched }\end{array}$ \\
\hline Heart Failure & $171(4.3)$ & $423(2.4)$ & & & $\begin{array}{l}160 \\
(4.8)\end{array}$ & $154(4.6)$ \\
\hline Hemorrhage & $163(4.1)$ & 1879 ( 10.8) & & & $\begin{array}{l}163 \\
(4.8)\end{array}$ & $126(3.7)$ \\
\hline Infection & $74(1.9)$ & 487 ( 2.8$)$ & & & $74(2.2)$ & $74(2.2)$ \\
\hline Kidney Failure & $22(0.6)$ & $110(0.6)$ & & & $19(0.6)$ & $13(0.4)$ \\
\hline Liver Disease & $6(0.2)$ & 599 ( 3.4) & & & $6(0.2)$ & $5(0.1)$ \\
\hline MISC & $780(19.5)$ & 7034 ( 40.3) & & & $\begin{array}{l}773 \\
(23.0)\end{array}$ & 738 (21.9) \\
\hline $\begin{array}{l}\text { Myocardial } \\
\text { Infarction }\end{array}$ & $\begin{array}{l}1416 \\
(35.4)\end{array}$ & 1579 ( 9.0) & & & $\begin{array}{l}971 \\
(28.9)\end{array}$ & $\begin{array}{l}1102 \\
(32.8)\end{array}$ \\
\hline Pancreatitis & $10(0.3)$ & $166(1.0)$ & & & $10(0.3)$ & $10(0.3)$ \\
\hline Pneumonia & $99(2.5)$ & $730(4.2)$ & & & $98(2.9)$ & $96(2.9)$ \\
\hline $\begin{array}{l}\text { Respiratory } \\
\text { Failure }\end{array}$ & $238(6.0)$ & 991 ( 5.7) & & & $\begin{array}{l}235 \\
(7.0)\end{array}$ & $201(6.0)$ \\
\hline Sepsis & 247 ( 6.2) & $1370(7.9)$ & & & $\begin{array}{l}243 \\
(7.2)\end{array}$ & $225(6.7)$ \\
\hline Tachycardia & $23(0.6)$ & $48(0.3)$ & & & $18(0.5)$ & $16(0.5)$ \\
\hline Vavular Disease & $544(13.6)$ & $878(5.0)$ & & & $\begin{array}{l}403 \\
(12.0)\end{array}$ & $421(12.5)$ \\
\hline \multicolumn{7}{|c|}{$\begin{array}{l}\text { Continuous variables are presented as mean (standard deviation), categorical as frequency } \\
\text { (percentage). T-test was used to compare statin recipients vs non-statin for continuous variables, } \\
\text { Fisher' s exact test for categorical variables. Standardized differences (SD) are defined as the } \\
\text { difference in means, proportions or ranks divided by the mutual standard deviation. (*)variables were } \\
\text { used for the calculation of propensity scores.Abbreviations:LOS: length of stay; OASIS: Oxford Acute } \\
\text { Severity of Illness Score; SOFA: Sequential Organ Failure Assessment; SAPSII: Simplified Acute } \\
\text { Physiology Score II. }\end{array}$} \\
\hline
\end{tabular}

\section{The primary outcome and doubly robust analysis}

The Kaplan-Meier analysis showed that statin users had a better 28-day and in hospital survival curve than the non-users in both unmatched and matched cohorts (Fig. 2). The univariate Cox model showed that the use of statins before or during ventilation was associated with an improved 28-day survival in both the overall cohort (HR $0.8595 \% \mathrm{Cl} 0.80 \sim 0.90$ Fig. 2A) and the matched cohort (HR $0.7995 \% \mathrm{Cl}$ $0.73 \sim 0.85$ Fig. $2 B$ ). In the multivariate Cox model, the use of statins was associated with a beneficial effect on 28-day survival in the unmatched cohort (HR $0.7395 \% \mathrm{Cl} 0.68 \sim 0.77$ Table 2 ) and the matched cohort (HR $0.7395 \% \mathrm{Cl} 0.68 \sim 0.78$ Table 2). Under the doubly robust estimation framework, an univariate and a multivariate regression model was developed to adjust for these unbalanced covariates on the 
weighted cohort (Table 2). The univariate model showed the HR of statins was 0.85 ( $95 \% \mathrm{Cl} 0.80 \sim 0.90$, $\mathrm{p}<0.01)$ where in the multivariate weighted model it was $0.85(95 \% \mathrm{Cl} 0.82 \sim 0.88, \mathrm{p}<0.01$, Table 2$)$.

Table 2

Five different models of primary outcome analysis

\begin{tabular}{|llc|}
\hline & HR & $\mathbf{p}$ \\
\hline Univariate COX model & $0.85[0.80,0.90]$ & $<0.01$ \\
\hline Univariate in propensity matched cohorts & $0.79[0.73,0.85]$ & $<0.01$ \\
\hline Propensity score IPW & $0.91[0.88,0.94]$ & $<0.01$ \\
\hline Multivariate COX model & $0.73[0.68,0.77]$ & $<0.01$ \\
\hline Doubly robust with multivariate & $0.85[0.82,0.88]$ & $<0.01$ \\
\hline $\begin{array}{l}\text { Model1, COX model with only the statin in the COX model; Model2, COX model with only statin in the } \\
\text { COX equation after propensity matching; Model3, after propensity matching, the univariate COX } \\
\text { model was weighted with propensity score; Model4, a multivariate COX model with all the covariates; } \\
\text { Model5, Doubly robust analysis with multivariate and weighted with the propensity score. }\end{array}$ \\
\hline
\end{tabular}

\section{Secondary outcomes and plasma biomarkers}

The secondary outcomes included VFDs and ICU free days. After matching, statin use was associated with more VFDs $(14.93 \pm 13.11$ vs $12.06 \pm 13.26)$ and more ICU free days $(13.41 \pm 12.14$ vs. $10.86 \pm 12.19)$ (Table 3). We analyzed the effect of statin on survival in patients with different lengths of stay in the matched cohort. Statin use was associated with improved 60-day (HR 0.81, 95\% Cl 0.75 0.86, $\mathrm{p}<0.01$ ), 90-day(HR 0.81, 95\% Cl $0.76 \sim 0.87, \mathrm{p}<0.01$ ) and in-hospital survival (HR 0.81, 95\% Cl $0.76 \sim 0.87, \mathrm{p}<$ 0.01 ) in the matched cohort (Table 3 ).

Table 3

The secondary outcomes.

\begin{tabular}{|llll|}
\hline & Non-statin & Statin & p \\
\hline $\mathrm{n}$ & 3363 & 3363 & \\
\hline VFD28 (mean (SD)) & $12.06(13.26)$ & $14.93(13.11)$ & $<0.01$ \\
\hline ICU-free28 (mean (SD)) & $10.86(12.19)$ & $13.41(12.14)$ & $<0.01$ \\
\hline Survival & & HR & $\mathrm{p}$ \\
\hline 60-day survive & 1 & $0.81[0.75,0.86]$ & $<0.01$ \\
\hline 90-day survive & 1 & $0.81[0.76,0.87]$ & $<0.01$ \\
\hline In hospital & 1 & $0.81[0.76,0.87]$ & $<0.01$ \\
\hline
\end{tabular}

VFD28: ventilator free days in 28 days; ICU-Free28: ICU free days in 28 days. 
Regarding the biomarkers, we found no large differences in the matched cohort. Additionally, we found that statin use was not associated with higher aspartate aminotransferase (AST) or alanine aminotransferase (ALT) levels after matching (Fig. 3). The WBC and neutrophils were significantly lower in the statin cohorts. However, the creatinine was slightly higher in the statin group.

\section{Sensitivity and subgroup analysis}

We further analyzed the different statins and their effect on patients' 28-day survival. All the different kinds of statins were found to be associated improved 28-day survival of the patients. We compared users of different types of statins to nonusers by COX regression model. In the COX model, all statins improved the survival of ventilated patients. Other statin groiup seemed to be associated with the best

outcome (HR $0.4895 \% \mathrm{Cl} 0.35 \sim 0.65$ ), followed by rosuvastatin (HR $0.5995 \% \mathrm{Cl} 0.41 \sim 0.85$ ), pravastatin (HR $0.6595 \% \mathrm{Cl} 0.49 \sim 0.87$ ) and simvastatin (HR $0.7295 \% \mathrm{Cl} 0.64 \sim 0.81$ ). The least-effective statin was atorvastatin, which was associated with a decreased 28-day mortality (HR $0.8495 \% \mathrm{Cl} 0.77 \sim 0.92)$. After adjusting for confounders, all the statins still resulted in significant improvement in survival compared to nonuse based on this survival function (Fig. 4A).

In the subgroup analysis, we found that statins had a protective effect in most of the subgroups but not in individuals with obesity. More importantly, statin use showed no effect in inflammation-related groups, such as the septicemia, respiratory failure and pneumonia subgroups (Fig. 4B). In the current study, patients with coronary disease accounted for a large proportion of the sample. However, in the noncoronary artery disease subgroup, we also found that statin use was associated with reduced mortality.

\section{Discussion}

This study revealed that statin use was associated with improved 28-day survival and in-hospital survival. All kinds of statins showed reduced HRs. This evidence might indicate the protective effect of statins in ventilation patients. In the subgroup analyses, we found that statins had a protective effect in most of the subgroups but not in the septicemia, acute respiratory failure or pneumonia subgroups. All these results might explain the protective effect of statins.

In addition to lowering cholesterol, statins exert pleiotropic effects [3-6] such as anti-inflammatory, antioxidant, and immunomodulatory effects, especially in the context of pulmonary disorders [30]. Statins may reduce COPD exacerbation [31]. Statins have also been suggested to be effective in patients with acute lung injury or acute respiratory distress syndrome in some observational studies [32, 33]. These clinical effects may be mediated by a reduction in pulmonary and systemic inflammation. Simvastatin decreased bronchoalveolar lavage IL-8 by 2.5 -fold $(P=0.04)$ [34]. Statins also showed a protective effect against sepsis. When compared with nonusers, simvastatin ( $\mathrm{HR}, 0.72 ; 95 \% \mathrm{Cl}, 0.58-0.90)$ and atorvastatin (HR, $0.78 ; 95 \% \mathrm{Cl}, 0.68-0.90)$ users had improved 30-day survival [10]. The current study examined the anti-inflammatory effect of statins from a ventilation patient cohort, which has not been 
previously reported. A recent study showed that infections in older adults were associated with prolonged, impaired neutrophil migration. Simvastatin improves neutrophil migration in vivo in healthy individuals and in vitro in milder infective events but not in severe sepsis, supporting its potential utility as an early intervention during pulmonary infections $[35,36]$. Lung injury remains one of the major complications of mechanical ventilation in the ICU. This injury could result from an altered host immune response after mechanical stretch [37]. Using statin therapy to protect patients with lung injury could therefore be a reasonable strategy, as these drugs could abate the host inflammatory response to infection [13], especially within the lungs [3].

Animal models may have further explained the protective effect of statins against lung injury. Statins increase glucocorticoid receptor expression in alveolar macrophages and downregulate NF-KB activation associated with the increased number of alveolar macrophages [12]. Two other animal models of lung injury induced by mechanical ventilation also support these findings [38, 39]. The protective effect may be due to the anti-inflammatory effect of statins. Prior statin use was associated with a lower baseline IL6 concentration, and continuation of atorvastatin treatment in this cohort was associated with improved survival [40]. Statins have been shown to reduce vascular leakage and inflammation in animal models of lung injury [41]. Statins may also attenuate lung injury by downregulating the expression of inflammatory cytokines $[42,43]$. Our previous study revealed the lung-protective effect of statins caused by the reduction of inflammatory cell infiltration [44]. Additionally, statins may have direct antibacterial effects and modulate bacterial virulence [45-47]. Sarah et al. showed that prior exposure to physiological nanomolar serum concentrations of simvastatin confers significant cellular resistance to the cytotoxicity of pneumolysin, which revealed how statins contribute to the reduced pathology observed in the context of pneumonia and other bacterial infections [48].

However, some randomized controlled studies have come to the opposite conclusion[14-18]. In a randomized controlled trial (RCT) with a follow-up of over one year, there was no significant difference in cumulative survival between the rosuvastatin and placebo groups ( $58 \%$ vs $61 \%$; $=0.377$ ) [49]. Simvastatin therapy was not significantly associated with the difference between the study groups in mortality at 28 days $(22.0 \%$ and $26.8 \% ; P=0.23)$ among patients with ARDS [18]. The contradictory results may be due to different study population. These RCTs have focused on patients with severe conditions such as severe ARDS and sepsis. Similarly, the subgroup analyses in this study showed that statins had no efficacy in the context of pneumonia, septicemia or acute respiratory failure. However, this difference might be explained by the fact that statins may exert a protective effect only for mild-to-moderate pulmonary infectious disease. From this point of view, the subgroup analyses explained why evidence of the efficacy of statins against sepsis and ARDS is controversial. Sapey stated that statins may improve neutrophil migration and may have protective effects in milder infective events but not in severe sepsis or ARDS [35]. The underlying reason for this controversial evidence might be that statins may have immune modulatory effects in only milder diseases instead of in intensive inflammatory diseases such as ARDS. However, we might need new RCTs exploring the effects on mild infectious diseases such as ventilationinduced lung injury to prove this hypothesis. 
Several limitations must be disclosed in the current study. The main limitation of this study was that the observational nature without randomization precludes a definite conclusion regarding statin benefits. However, a randomized controlled trial on the effect of long-term statin treatment on the outcome of patients on ventilation would require many participants. To explore the effect of statins on patients on ventilation, observational data may currently remain the best available evidence. Second, because of the retrospective design of this study, patient selection bias may be inevitable. Third, the missing data of potential confounders was a limitation that could not be overcome. Our findings should thus be interpreted with caution.. Regarding the host response, the assessment of inflammatory cytokines might provide different insights. The relationships of the dose and treatment duration of statins with survival were not analyzed here because we included different types of statins, and the doses of the different statins were not comparable. Finally, we included ventilation patients with different diagnoses. Even with subgroup analyses, we cannot conclude that statins are specifically effective in a specific population. Further studies in different populations or RCTs are needed to validate our findings.

\section{Conclusions}

Our study suggests that the use of statins may be associated with reduced mortality in ventilated patients. Statins may exert protective effect only in mild-to-moderate patients instead of critical inflammatory ones.

\section{Abbreviations}

MIMIC-III: Medical Information Mart for Intensive Care III; LOS: length of stay; length of stay; VFDs: ventilator-free days; ARDS: acute respiratory distress syndrome; ICU: intensive care unit ; IRB: institutional review board; SAPSII: Simplified Acute Physiology Score II; Cl: confidence interval (CI); SD: standard deviations ; HR: Hazard Ratio; AST: aminotransferase; ALT: alanine aminotransferase; RCT: randomized controlled trial; WBC: white blood cell.

\section{Declarations}

Ethic approval and consent to participate: The requirement for institutional review board (IRB) approval from our institution was exempted because MIMIC-III is a third-party anonymized publicly available database with pre-existing IRB approval. The consent to participate was waived because the retrospective design of the study.

Consent for publication: Not applicable.

Availability of data and material: The data can be available by contacting the corresponding author Jiang Du by email gowindj@163.com. The source codes for statistical analyses can be found at https://github.com/gowindj/vent_statin[50]. The source codes for postgreSQL can be found at https://github.com/gowindj/public[51]. 
Competing interest: All the authors declare that they have no potential financial or ethical conflicts of interest regarding the contents of this manuscript.

Funding: Sponsored by the Interdisciplinary Program of Shanghai Jiao Tong University (Project Number 06N1801046). Additionally sponsored by Clinical Research Innovation Plan of Shanghai General Hospital (Project Number CTCCR-2018C10)

Authors' contributions: CD write the manuscript collect the data from MIMIC database, washed the data and did the statistical analysis. WL contributed to the data collecting work and writing of the manuscript. $\mathrm{ZH}$ contributed to the data collecting of this work, statistical analysis and writing of this manuscript. LQ contributed to collect the data from MIMIC database. MC contributed to data collecting and data analysis of this work. XY contributed to the data collecting and analysis of this work. HY contributed to the data collecting work. GF contributed to the data collecting work. XJ contributed to the data collecting and literature research of this work. KM contributed to the statistic work of this study. TR contributed to the study design and paper revising. ZY contributed to the data collecting of this work. WR designed this research, revised the manuscript and analyzed the data. Du, Jiang designed this research, write the manuscript, analyzed the data and drew all the figures in this study. Jiang Du takes responsibility for (is the guarantor of) the content of the manuscript, including the data and analysis ( Original Research).

Acknowledgments: Not applicable.

\section{References}

1. Willeit P, Ridker PM, Nestel PJ, Simes J, Tonkin AM, Pedersen TR et al: Baseline and on-statin treatment lipoprotein(a) levels for prediction of cardiovascular events: indiv idual patient-data metaanalysis of statin outcome trials. Lancet 2018, 392(10155):1311-1320.

2. Cohen JS: Statin therapy after stroke or transient ischemic attack. N Engl J Med 2006, 355(22):2368; author reply 2370-2361.

3. Krishna RK, Issa O, Saha D, Macedo FY, Correal B, Santana O: Pleiotropic effects of the 3-hydroxy-3methylglutaryl-CoA reductase inhibitors in pulmonary diseases: a comprehensive review. Pulmonary pharmacology \& therapeutics 2015, 30(134-140.

4. Liao JK, Oesterle A: The Pleiotropic Effects of Statins - From Coronary Artery Disease and Stroke to Atrial Fibrillation a nd Ventricular Tachyarrhythmia. Curr Vasc Pharmacol 2018.

5. Mihos CG, Santana O: Pleiotropic effects of the HMG-CoA reductase inhibitors. Int J Gen Med 2011, 4(261-271.

6. Rohilla A, Rohilla S, Kumar A, Khan MU, Deep A: Pleiotropic effects of statins: A boulevard to cardioprotection. Arabian Journal of Chemistry 2016, 9(S21-S27.

7. Terblanche M, Almog Y, Rosenson RS, Smith TS, Hackam DG: Statins and sepsis: multiple modifications at multiple levels. Lancet Infect Dis 2007, 7(5):358-368. 
8. Nielsen AG, Nielsen RB, Riis AH, Johnsen SP, Sørensen HT, Thomsen RW: The impact of statin use on pneumonia risk and outcome: a combined population-based case-control and cohort study. Critical care 2012, 16(4):R122.

9. Al Harbi SA, Tamim HM, Arabi YM: Association between statin therapy and outcomes in critically ill patients: a nested cohort study. BMC Clin Pharmacol 2011, 11(12.

10. Lee CC, Lee MG, Hsu TC, Porta L, Chang SS, Yo CH et al: A Population-Based Cohort Study on the Drug-Specific Effect of Statins on Sepsis Outcome. Chest 2017.

11. Wan YD, Sun TW, Kan QC, Guan FX, Zhang SG: Effect of statin therapy on mortality from infection and sepsis: a meta-analysis of randomized and ob servational studies. Crit Care 2014, 18(2):R71.

12. Takano K, Yamamoto S, Tomita K, Takashina M, Yokoo H, Matsuda N et al: Successful treatment of acute lung injury with pitavastatin in septic mice: potential role of glucoco rticoid receptor expression in alveolar macrophages. J Pharmacol Exp Ther 2011, 336(2):381-390.

13. Tleyjeh IM, Kashour T, Hakim FA, Zimmerman VA, Erwin PJ, Sutton AJ et al: Statins for the prevention and treatment of infections: a systematic review and meta-analysis. Arch Intern Med 2009, 169(18):1658-1667.

14. Kruger PS, Harward ML, Jones MA, Joyce CJ, Kostner KM, Roberts MS et al: Continuation of Statin Therapy in Patients with Presumed Infection. American Journal of Respiratory and Critical Care Medicine 2011, 183(6):774-781.

15. Singh RK, Agarwal V, Baronia AK, Kumar S, Poddar B, Azim A: The Effects of Atorvastatin on Inflammatory Responses and Mortality in Septic Shock: A Single-center, Randomized Controlled Trial. Indian J Crit Care Med 2017, 21(10):646-654.

16. Nagendran M, McAuley DF, Kruger PS, Papazian L, Truwit JD, Laffey JG et al: Statin therapy for acute respiratory distress syndrome: an individual patient data meta-analysis of $r$ andomised clinical trials. Intensive Care Med 2017, 43(5):663-671.

17. National Heart L, Blood Institute ACTN, Truwit JD, Bernard GR, Steingrub J, Matthay MA et al: Rosuvastatin for sepsis-associated acute respiratory distress syndrome. N Engl J Med 2014, 370(23):2191-2200.

18. McAuley DF, Laffey JG, O'Kane CM, Perkins GD, Mullan B, Trinder TJ et al: Simvastatin in the acute respiratory distress syndrome. The New England journal of medicine 2014, 371(18):1695-1703.

19. Chen M, Ji M, Si X: The effects of statin therapy on mortality in patients with sepsis: A meta-analysis of randomized trials. Medicine (Baltimore) 2018, 97(31):e11578.

20. Oh TK, Song IA, Choi S: Prior statin therapy and mortality among critically ill patients: a systemic review and meta-analysis of cohort studies. Ann Trans/ Med 2020, 8(6):396.

21. !!! INVALID CITATION !!! \{Kyu Oh, 2019 \#64\}.

22. Wunsch H, Linde-Zwirble WT, Angus DC, Hartman ME, Milbrandt EB, Kahn JM: The epidemiology of mechanical ventilation use in the United States. Crit Care Med 2010, 38(10):1947-1953.

23. Tobin MJ: Mechanical ventilation. New England Journal of Medicine 1994, 330(15):1056-1061. 
24. Johnson AE, Pollard TJ, Shen L, Lehman LW, Feng M, Ghassemi M et al: MIMIC-III, a freely accessible critical care database. Sci Data 2016, 3(160035.

25. Mccaffrey DF, Griffin BA, Almirall D, Slaughter ME, Burgette LF: A Tutorial on Propensity Score Estimation for Multiple Treatments Using Generalized Boosted Models. Statistics in Medicine 2013, 32(19):3388-3414.

26. Feng M, McSparron JI, Kien DT, Stone DJ, Roberts DH, Schwartzstein RM et al: Transthoracic echocardiography and mortality in sepsis: analysis of the MIMIC-III database. Intensive Care Med 2018, 44(6):884-892.

27. Cole SR, Hernan MA: Constructing inverse probability weights for marginal structural models. Am J Epidemiol 2008, 168(6):656-664.

28. Kamat SA, Gandhi SK, Davidson M: Comparative effectiveness of rosuvastatin versus other statin therapies in patients at increased risk of failure to achieve low-density lipoprotein goals. Curr Med Res Opin 2007, 23(5):1121-1130.

29. Masadeh M, Mhaidat N, Alzoubi K, Al-Azzam S, Alnasser Z: Antibacterial activity of statins: a comparative study of atorvastatin, simvastatin, and rosuvastatin. Ann Clin Microbiol Antimicrob 2012, 11(13.

30. Chopra V, Flanders SA: Does Statin Use Improve Pneumonia Outcomes? Chest 2009, 136(5):13811388.

31. Blamoun Al, Batty GN, DeBari VA, Rashid AO, Sheikh M, Khan MA: Statins may reduce episodes of exacerbation and the requirement for intubation in patients with COPD: evidence from a retrospective cohort study. International journal of clinical practice 2008, 62(9):1373-1378.

32. O'Neal HR, Koyama T, Koehler EAS, Siew E, Curtis BR, Fremont RD et al: Prehospital statin and aspirin use and the prevalence of severe sepsis and acute lung injury/acute respiratory distress syndrome. Critical Care Medicine 2011, 39(6):1343-1350.

33. Mansur A, Steinau M, Popov AF, Ghadimi M, Beissbarth T, Bauer M et al: Impact of statin therapy on mortality in patients with sepsis-associated acute respiratory distress syndrome (ARDS) depends on ARDS severity: a prospective observational cohort study. Bmc Med 2015, 13(

34. Craig TR, Duffy MJ, Shyamsundar M, McDowell C, O'Kane CM, Elborn JS et al: A randomized clinical trial of hydroxymethylglutaryl- coenzyme a reductase inhibition for acute lung injury (The HARP Study). American journal of respiratory and critical care medicine 2011, 183(5):620-626.

35. Sapey E, Patel JM, Greenwood HL, Walton GM, Hazeldine J, Sadhra C et al: Pulmonary Infections in the Elderly Lead to Impaired Neutrophil Targeting, Which Is Improved by Simvastatin. Am J Respir Crit Care Med 2017, 196(10):1325-1336.

36. Sapey E, Patel JM, Greenwood H, Walton GM, Grudzinska F, Parekh D et al: Simvastatin Improves Neutrophil Function and Clinical Outcomes in Pneumonia: a Pilot Randomised Contr olled Trial. Am J Respir Crit Care Med 2019.

37. Charles PE, Tissieres P, Barbar SD, Croisier D, Dufour J, Dunn-Siegrist I et al: Mild-stretch mechanical ventilation upregulates toll-like receptor 2 and sensitizes the lung to bacterial lipopeptide. Crit Care 
2011, 15(4):R181.

38. $M \circledR \pi$ ller HC, Hellwig K, Rosseau S, Tschernig T, Schmiedl A, Gutbier B et al: Simvastatin attenuates ventilator-induced lung injury in mice. Crit Care 2010, 14(4):R143.

39. Siempos, II, Maniatis NA, Kopterides P, Magkou C, Glynos C, Roussos C et al: Pretreatment with atorvastatin attenuates lung injury caused by high-stretch mechanical ventilation i $\mathrm{n}$ an isolated rabbit lung model. Crit Care Med 2010, 38(5):1321-1328.

40. Kruger P, Bailey M, Bellomo R, Cooper DJ, Harward M, Higgins A et al: A multicenter randomized trial of atorvastatin therapy in intensive care patients with severe sepsis. Medicine 2013, 187(7):743-750.

41. Jacobson JR, Barnard JW, Grigoryev DN, Ma SF, Tuder RM, Garcia JG: Simvastatin attenuates vascular leak and inflammation in murine inflammatory lung injury. American Journal of Physiology Lung Cellular \& Molecular Physiology 2005, 288(6):1026-1032.

42. Melo AC, Valenca SS, Gitirana LB, Santos JC, Ribeiro ML, Machado MN et al: Redox markers and inflammation are differentially affected by atorvastatin, pravastatin or simvastatin administered before endotoxin-induced acute lung injury. International immunopharmacology 2013, 17(1):57-64.

43. Shyamsundar M, Mckeown STW, O'Kane CM, Craig TR, Brown V, Thickett DR et al: Simvastatin Decreases Lipopolysaccharide-induced Pulmonary Inflammation in Healthy Volunteers. American Journal of Respiratory \& Critical Care Medicine 2009, 179(12):1107-1114.

44. Du J, Zhu Y, Meng X, Xie H, Wang J, Zhou Z et al: Atorvastatin attenuates paraquat poisoninginduced epithelial-mesenchymal transition via downregulating hypoxia-inducible factor-1 alpha. Life Sciences.

45. Hennessy E, Adams C, Reen FJ, O'Gara F: Is There Potential for Repurposing Statins as Novel Antimicrobials? Antimicrob Agents Chemother 2016, 60(9):5111-5121.

46. Ting M, Whitaker EJ, Albandar JM: Systematic review of the in vitro effects of statins on oral and perioral microorganisms. Eur J Oral Sci 2016, 124(1):4-10.

47. Ribeiro NQ, Costa MC, Magalh?es TFF, Carneiro HCS, Oliveira LV, Fontes ACL et al: Atorvastatin as a promising anticryptococcal agent. Int J Antimicrob Agents 2017, 49(6):695-702.

48. Statt S, Ruan JW, Hung LY, Chang CY, Huang CT, Lim JH et al: Statin-conferred enhanced cellular resistance against bacterial pore-forming toxins in airway epithelial cells. Am J Respir Cell Mol Biol 2015, 53(5):689-702.

49. Dinglas VD, Hopkins RO, Wozniak AW, Hough CL, Morris PE, Jackson JC et al: One-year outcomes of rosuvastatin versus placebo in sepsis-associated acute respiratory distress syndrome: prospective follow-up of SAILS randomised trial. Thorax 2016, 71(5):401-410.

50. Du J: R codes: https://github.com/gowindj/vent_statin; 2020

51. Du J: PostgreSQL: https://github.com/gowindj/public.; 2020

\section{Figures}




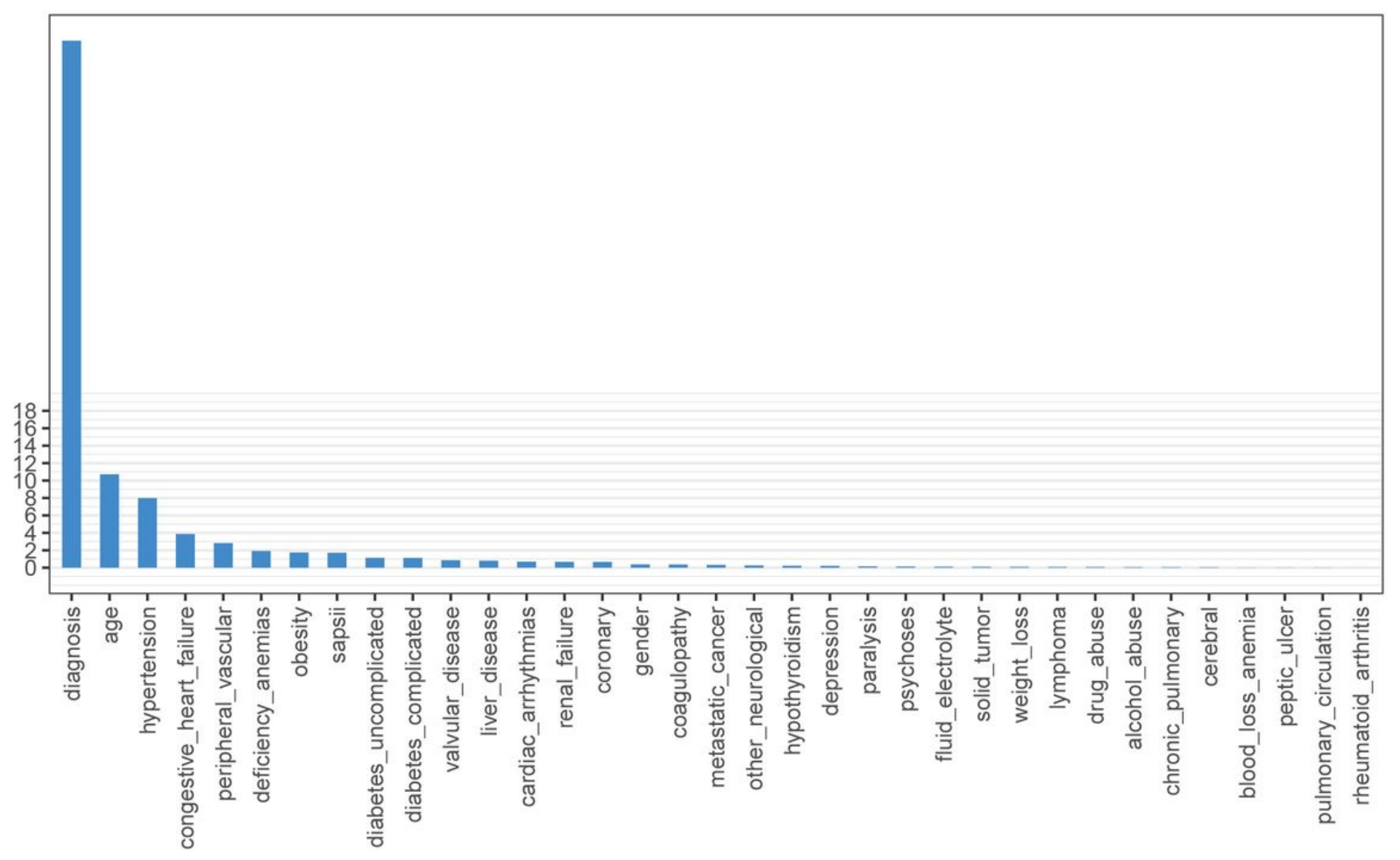

Figure 1

The plot of relative influence factor of covariates. The relative influence factor measures how discriminative the 35 covariates of the propensity score model are when predicting the likelihood of statin prescription. 
A 28 days survival of unmatched cohorts

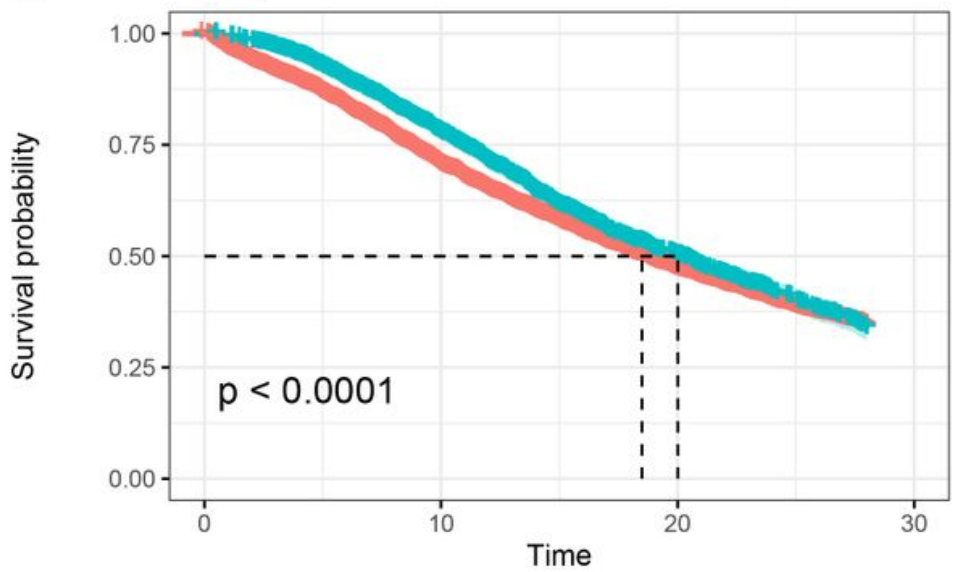

Number at risk

\begin{tabular}{|c|c|c|c|}
\hline Non statin- 7426 & 8489 & 3863 & 0 \\
\hline Statin -3998 & 1777 & 639 & 0 \\
\hline 0 & 10 & 20 & 30 \\
\hline
\end{tabular}

B
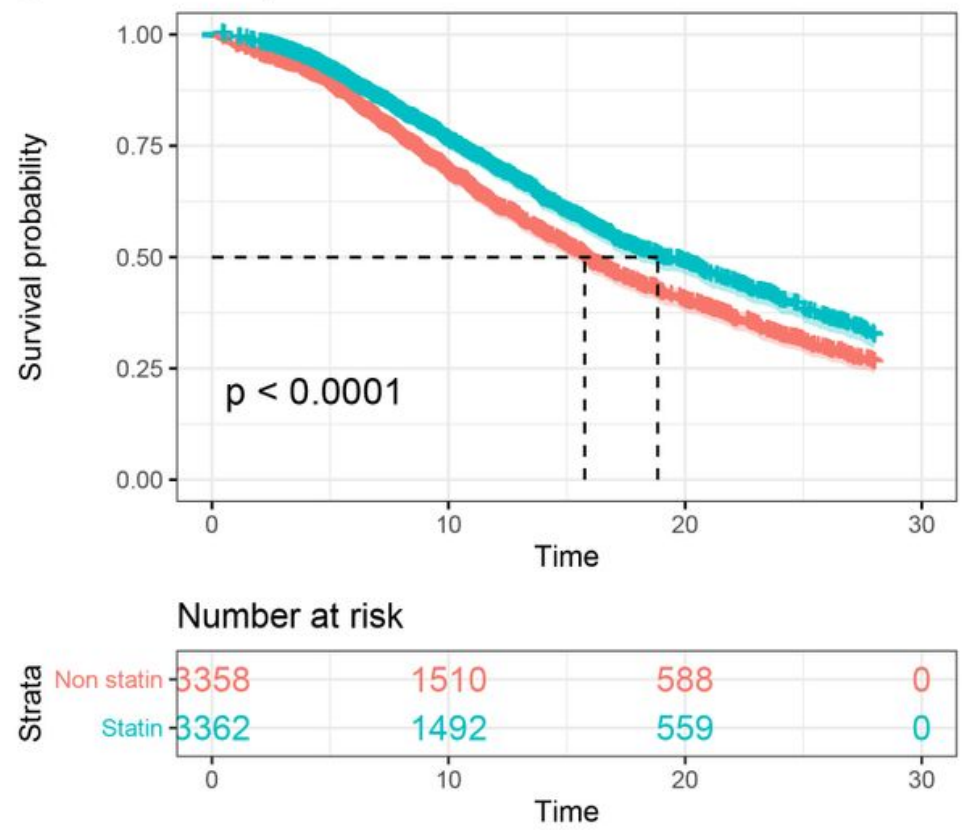

Figure 2

Kaplan-Meier curves for all-cause mortality in statin recipients vs. nonrecipients in the unmatched cohorts and propensity score-matched cohorts. / A: Statin use was associated with improved 28-day survival in the unmatched cohort (HR $0.8595 \% \mathrm{Cl} 0.80 \sim 0.90$ ); B: Statin use was associated with improved 28-day survival in the matched cohort (HR $0.7995 \% \mathrm{Cl} 0.73 \sim 0.85$ ).
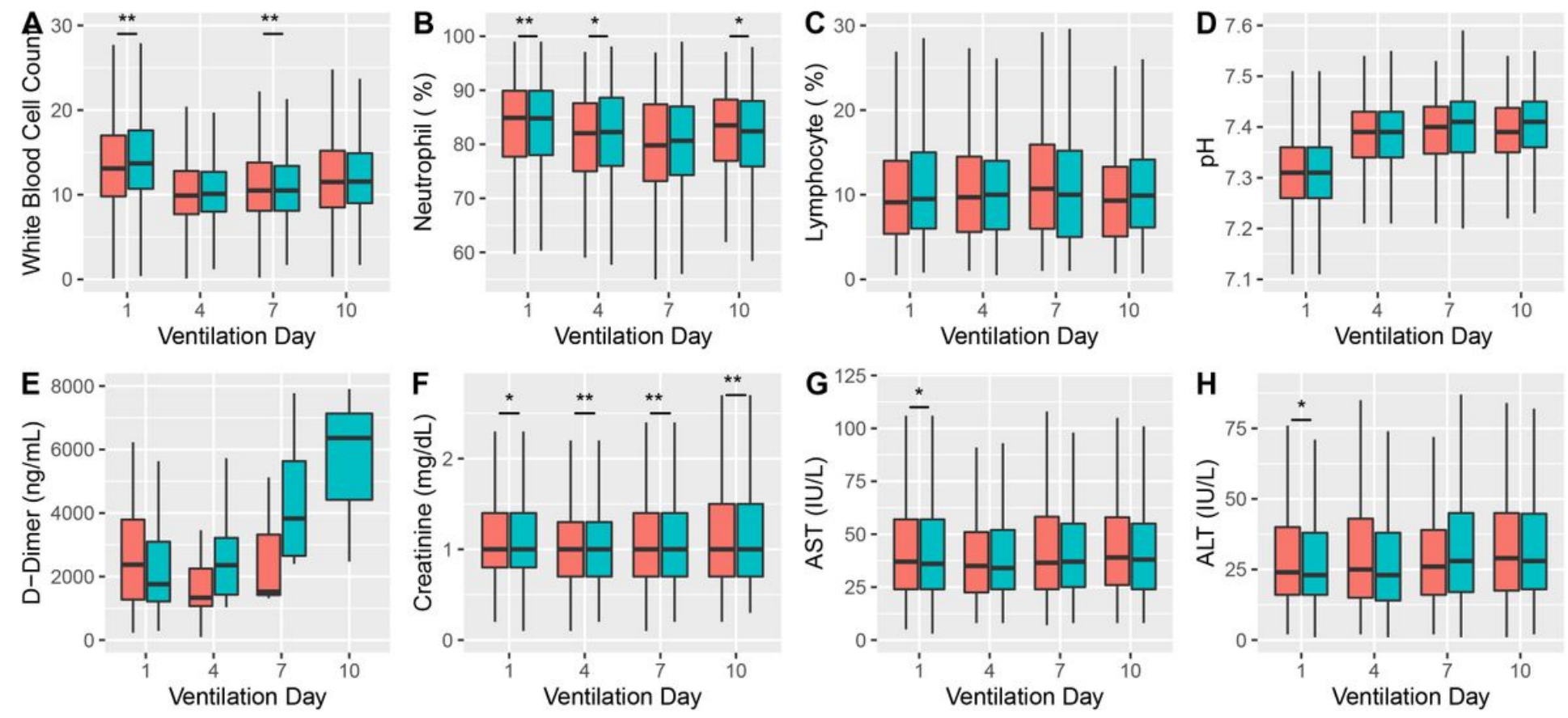

$$
\text { Statin 官 Non_Statin 它 Statin }
$$

Figure 3 
The plasma biomarkers during the first ten ventilation days./ Day 1 was the first day of ventilation; *: $p<0.05 ; * \star: p<0.01$.
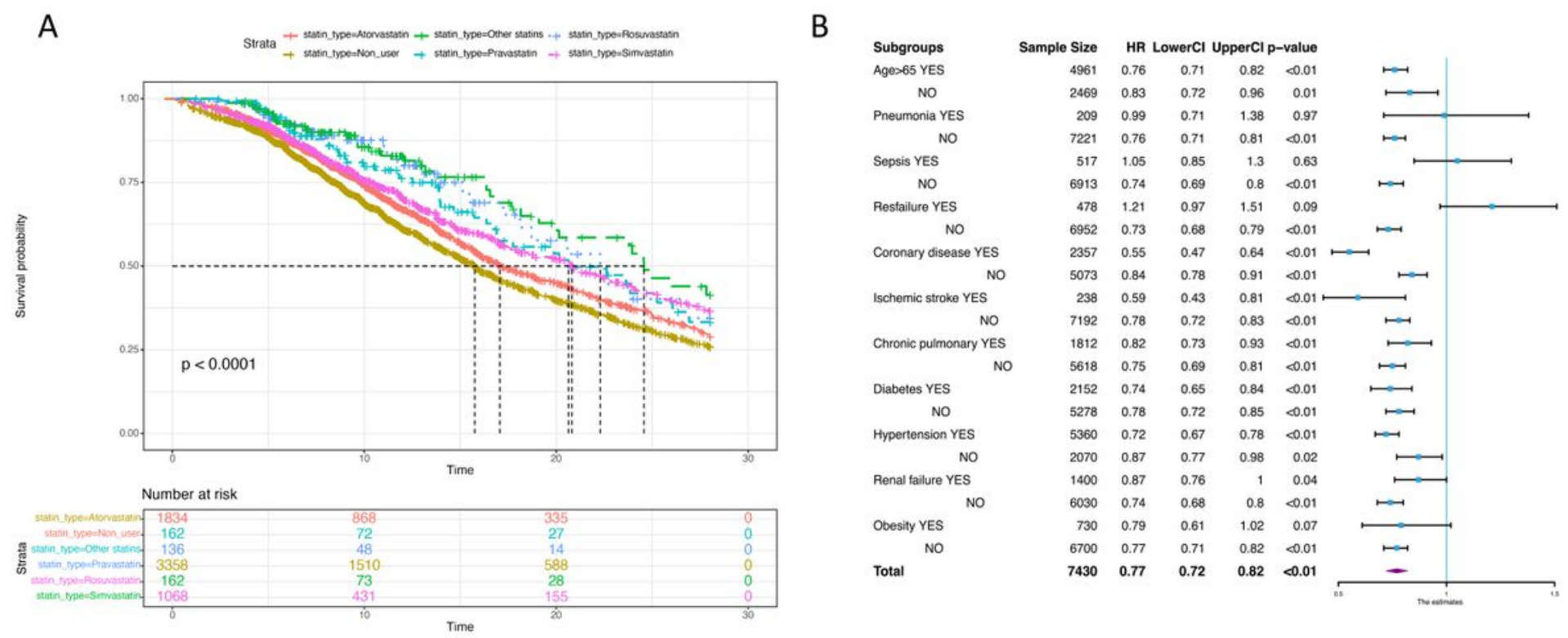

\section{Figure 4}

The plots of subtype analysis and subgroup analysis. /A:The different types of statin use and survival; B: The forest plot of statins in the 28-day survival subgroup analyses. 